\title{
Time-Domain EMI Measurements using a Low Cost Digitizer to Optimize the Total Measurement Time for a Test Receiver
}

\author{
Tom Hartman ${ }^{1}$, Roelof Grootjans ${ }^{1}$, Niek Moonen ${ }^{1}$, Frank Leferink ${ }^{1,2}$ \\ ${ }^{1}$ University of Twente, Enschede, The Netherlands \\ ${ }^{2}$ THALES Nederland B.V., Hengelo, The Netherlands \\ tom.hartman@utwente.nl
}

\begin{abstract}
By analyzing electromagnetic interference (EMI) based on its spectral components important time-domain information is lost. Conventional super-heterodyne frequency band stepping EMI test receivers have to use long dwell times for every frequency which makes the total measurement time too long. Often dwell times are set too short, causing time-varying interference, or cyclo-stationary interference, to be detected improperly. The concept of time-varying EMI is also not incorporated in standards, which are only based on frequency domain limits. To catch these time-varying interferences the receiver has to measure for at least one repetition period. Measuring many spectral components for at least the repetition of a cyclo-stationary signal causes detrimental measurement times. Time-domain electromagnetic interference (EMI) analyzers have been proposed to reduce these long measurement times, but remain expensive. To reduce costs the utilization of time-domain EMI measurements using a low cost digitizer is examined. A PicosScope in conjunction with Digital Signal Processing (DSP) is used to create the possibility to estimate the total measurement time of a conventional EMI receiver based on the dwell times. This can be used to optimize the total measurement time needed for the test receiver, while still complying to standards, resulting in reduced measurement times otherwise needed in expensive test labs. A short-time FastFourier transform (STFFT) is used to examine the interfering source in both frequency and time simultaneously. It was also shown that the conventional EMI receiver perceives certain time varying signals as continuous waves due to the spectral nature of this receiver.
\end{abstract}

\section{INTRODUCTION}

Analyzing electromagnetic interference (EMI) was traditionally done, i.e. decades ago, based on the spectral components of the interference. This was done because a timedomain analysis was still insufficiently accurate due to limitations of hardware, either due to the limited Analog to Digital Converter (ADC), sampling rate, memory or dynamic range [1]. The use of conventional super-heterodyne frequency band stepping EMI receivers overcame these issues by analyzing each frequency bin individually while sweeping through the spectrum. This however came with certain trade-offs, take for example how radiated emission measurements of magnetic fields are performed according to military standards (NRE01)

This project has received funding from the EMPIR programme co-financed by the Participating States and from the European Union's Horizon 2020 research and innovation programme. The results found reflect the author's view only. EURAMET is not responsible for any use that may be made of the information it contains.
[2]. These measurements are performed between $30 \mathrm{~Hz}$ and $100 \mathrm{kHz}$, with bandwidths starting at $10 \mathrm{~Hz}$ and steps of 5 $\mathrm{Hz}$. This gives rise to a lot of measurement steps that have to be taken. Also, due to the spectral analyzing nature of these receivers, time-domain information is lost. This timedomain information is important, because if an interference source is only apparent, for example, once every second, measuring for only half a second will not assure that the interference is measured correctly. This fact of an time-varying interference is also not incorporated in the standards, which are specified as a threshold over frequency [3]. To still notice the effect of the time-domain variation these receivers have to measure every frequency bin for a certain amount of time, with two parallel detectors. A well founded description of analyzing time-varying disturbances can be found in [4], where a simulation model is developed to mimic these types of detectors. The measurement time per frequency bin, the dwell time, is dependent on the time variation at that specific frequency. A lot of measurement steps and a minimum amount of measurement time per frequency bin result in a very long and detrimental measurement time, as has been shown in [5]. Additionally, having to do these measurements at many different positions around a large equipment under test (EUT) increases the total measurement time even more, for some systems this can even be as long as one week, resulting in very high costs [6]. To reduce these long and therefore expensive measurement times time-domain EMI analyzers became very popular, but remain expensive. Advantages [5], [7] and challenges [8], [9] of time-domain EMI analyzers have been discussed previously. Time-domain measurements are fully capable of accurate measurements and they have been shown to be compliant with standards, such as CISPR 16-1-1 [10], [11].

In this paper, a low cost multichannel digitizer known as a PicoScope is used in conjunction with digital signal processing (DSP) via a mathematical tool on a personal computer. The multichannel capabilities of the digitizer allow for many added benefits and possibilities for various situations [12], [13]. With this DSP on a commercial of the shelf (COTS) computer or laptop, it is proposed to quickly determine the minimum dwell times needed at every frequency bin. This can be used to make an estimation of the total measurement time needed for a 
conventional EMI receiver, after which the total measurement time can be optimized while still complying to the standards. This will reduce the detrimental and expensive measurement times otherwise needed in test chambers. The conventional EMI receiver used in this paper for verification is the Rohde \& Schwarz ESPI7 [14]. To accomplish the comparison, a short time fast Fourier transform (STFFT) is used to create a spectrogram that holds information in both the frequency and the time-domain. From this, the time-varying behaviour of frequency components is retrieved and analyzed by taking a fast Fourier transform (FFT) of the time slice and studying the lowest significant frequency. This will then be compared to the, time consuming, conventional measurements to obtain the dwell time, via parallel detectors, of a conventional EMI receiver.

This paper starts off in Section II with a description of the DSP on a PC and the constraints that arose when trying to comply to the standards when calculating the dwell times. This is then followed up with how a conventional EMI receiver perceives time-varying signals. Section III describes the measurement setup used to verify the processing on a PC. Section IV shows the results found via a conventional EMI receiver and the DSP on a PC, after which everything is wrapped up in the conclusion, Section V.

\section{THEORETICAL BACKGROUND}

In this section, several constrains for the spectrogram due to mimicking the conventional EMI test receiver that is used are addressed. An example of such a spectrogram can be seen in Fig. 1, where power per frequency is shown over frequency and time simultaneously. In this figure, one can clearly see the time varying aspect of some frequency components.

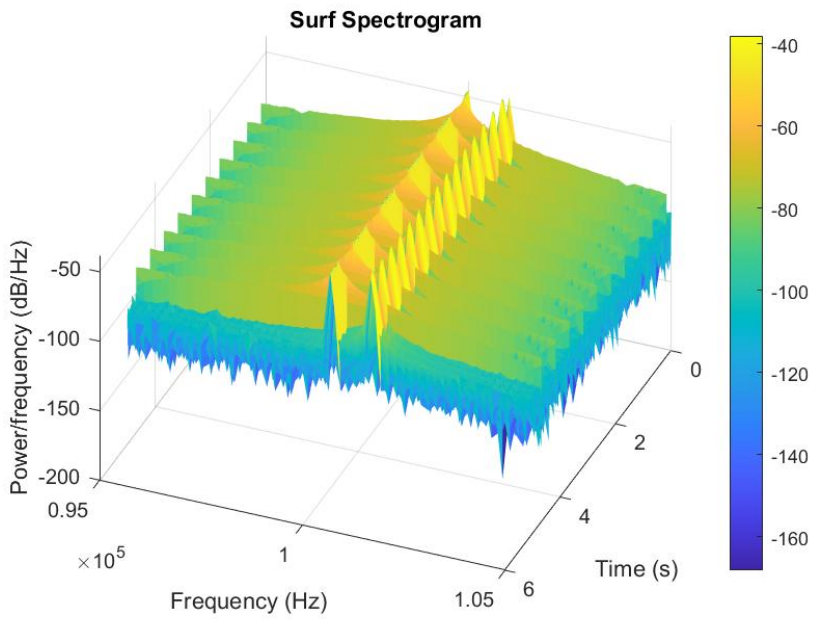

Fig. 1: Example spectrogram of a time variant signal

\section{A. Time Varying Signals}

A spectrogram is created by taking STFFTs of the input signal where the short time is defined by a window. This window is then shifted over time while still keeping a certain overlap, as to increase the time resolution. The dwell time is calculated by taking FFTs of the time slices at every frequency bin. From these FFTs, the lowest significant frequency is chosen which represents the inverse of the dwell time. The dwell time is defined as the minimum time needed by an EMI test receiver to accurately estimate a disturbance at a single frequency when the signal is time varying. In this section an example of a time varying signal is presented to inspect the time varying nature of EMI. This is followed up by an explanation of how the conventional EMI receiver filters the input signals and the influence it has on the perception of these time varying signals.

The signals used for the measurements are created with a signal generator and measured with the conventional EMI receiver parallel to the PicoScope to create a reliable and controlled measurement environment. Furthermore an actual switch mode power supply with a varying load is used later to represent real world situations. The time-domain data measured with the PicoScope is then processed by the algorithm to check if the same dwell times are found as the results given by the conventional EMI receiver. A representation of an electromagnetic disturbance is a Continuous wave $(\mathrm{CW})$ signal. To show the effect of a varying disturbance, this $\mathrm{CW}$ signal will be switched on and off completely. Another case that is realized is varying the $\mathrm{CW}$ smoothly from zero to full power and back. Both of these signals will be used as examples for controlled interference cases in the analysis and the measurements. An example of such a time varying interference is shown in Fig. 2. These signals are used because of their time varying nature and because it is easily produced and combined via a signal generator. While testing this signal some constraints arose about the definition of a time varying frequency component which will be explained further on.

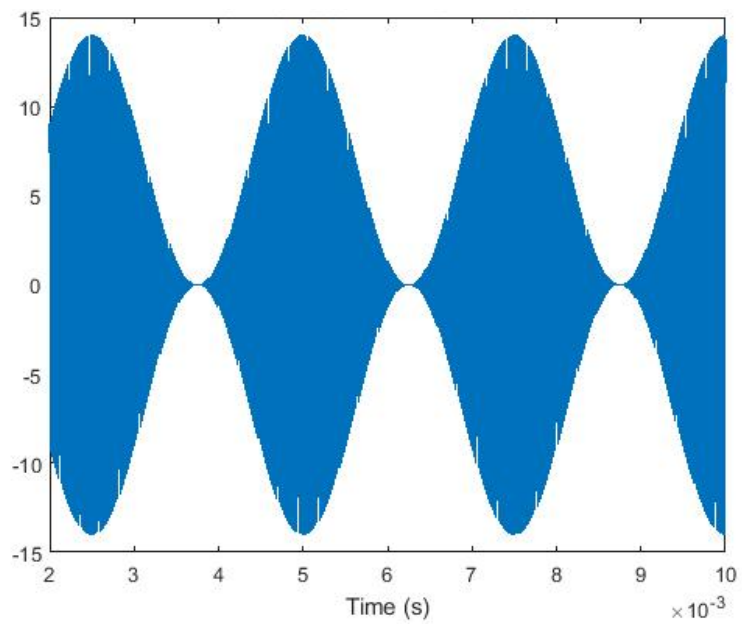

Fig. 2: Smooth varying interference at $50 \mathrm{kHz}$ with $400 \mathrm{~Hz}$ variation

\section{B. Frequency Resolution of the Spectrogram}

The first constraint is due to the fact that the DSP software, used on the time-domain signal obtained via a low cost 
TABLE I: CISPR-16-1-1 BANDWIDTHS FOR DIFFERENT MEASUREMENT RANGES

\begin{tabular}{|c|c|}
\hline Frequency Range & Detector Bandwidth \\
\hline $9 \mathrm{kHz}-150 \mathrm{kHz}$ (Band A) & $200 \mathrm{~Hz}$ \\
$150 \mathrm{kHz}-30 \mathrm{MHz}$ (Band B) & $9 \mathrm{kHz}$ \\
$30 \mathrm{MHz}-1000 \mathrm{MHz}$ (Band C \& D) & $120 \mathrm{kHz}$ \\
\hline
\end{tabular}

multichannel digitizer, is supposed to mimic a conventional EMI receiver. The CISPR16-1-1 is the standard for civil applications that defines different bandwidths for different frequency ranges as seen in Table I. From this table, the bandwidths can be found which can then be seen as the frequency resolution of the spectrogram, also known as the step size of the frequency axis. This resolution can be seen as the inverse of the length of the window, in seconds, or as the maximum attainable frequency divided by the number of bins created. The inverse of the window length can be written as:

$$
f_{\text {res }}=\frac{1}{T_{w}}=\frac{1}{\frac{N_{w}}{f s}}=\frac{f s}{N_{w}}
$$

where $f s$ is the sampling frequency, $N_{w}$ is the window size, and $f_{\text {res }}$ should conform with the specified bandwidths defined in Table I if one is interested in these ranges.

As previously mentioned, a conventional super-heterodyne EMI receiver analyzes EMI by its spectral components. It does so by going through all the frequencies within a range step by step. This can be seen as a filter bank shifting over the entire frequency range with a certain step size. Analyzing the frequency components in their individual filter banks separately raises some questions. Looking from the timedomain perspective Fig. 2 clearly resembles a signal with a high frequency component varying over time. Put even more strongly, the whole definition of this test case was to create a high frequency component which varies over time with a lower frequency component. This input signal is written down mathematically as:

$$
A(t) \cdot \cos \left(2 \pi f_{h} t\right),
$$

where $A(t)=1+\cos \left(2 \pi f_{l} t\right), f_{h}$ is the high frequency and $f_{l}$ is the low frequency. We know however that this can be seen as two individual frequency components around a high frequency component because:

$$
\cos (\alpha) \cdot \cos (\beta)=\frac{1}{2} \cdot[\cos (\alpha-\beta)+\cos (\alpha+\beta)]
$$

From this point on, the frequency resolution seen in equation (1), which can be seen as the width of a frequency bin of a filter bank, has a huge impact on how the signal is perceived. This is due to the fact that if this frequency bin width becomes smaller than the difference between adjacent frequency components it will not catch both frequency components simultaneously. The frequency components of electromagnetic interference of two independent interference sources could lie just outside a frequency bin, but be perceived by the system simultaneously as a time varying interference. As an example this is visualized in Fig. 3 and Fig. 4, where the frequency components are received in one frequency bin and in separate frequency bins respectively. The frequency bins are chosen to have a width of $200 \mathrm{~Hz}$, because this is the minimum width of the quasi-peak detector used in the conventional EMI receiver. If the frequency components are received in separate bins for the EMI receiver it is expected that it will perceive the signal as individual frequency components which are not varying over time, also known as individual continuous waves at different frequencies, which can also be mimicked via the STFFT by adjusting the frequency resolution if needed. The software in conjunction with the low cost digitizer can however also overcome this issue by specifying their bandwidth accordingly in the post-processing.

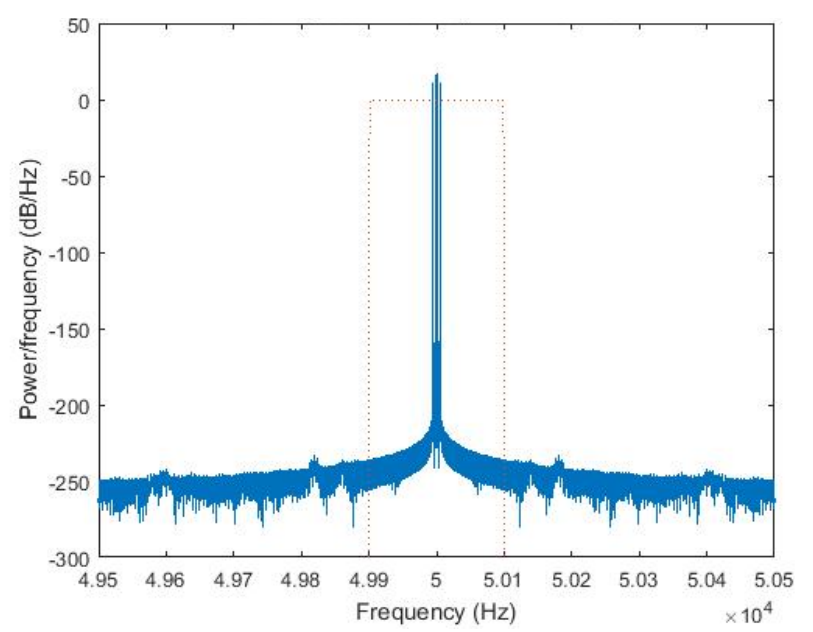

Fig. 3: Smooth varying interference with all frequency components in one filter bank

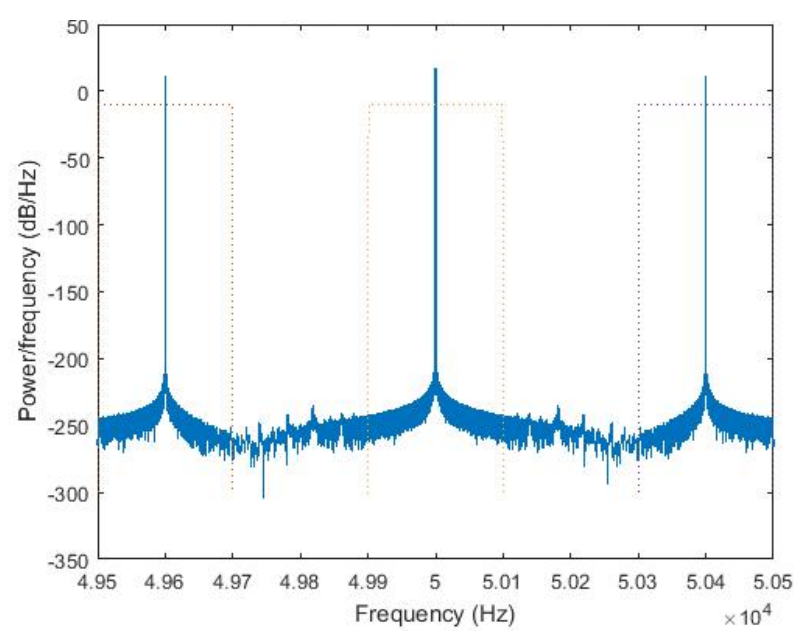

Fig. 4: Smooth varying interference with one frequency component per filter bank

\section{MEASUREMENT SETUP}

This section will describe the measurement setup that is used to show dwell time estimation using a conventional 
EMI test receiver and an oscilloscope for which a schematic representation is shown in Fig. 5. The test signal is generated with an arbitrary waveform generator (Teledyne LeCroy T3AFG120) [15], this allows full control over all the parameters of the signal and also what kind of switching is used. It allows the use of two independent channels and can also be configured to superimpose one channel onto the other. At the receiving end the traditional EMI test receiver (Rhode \& Schwarz ESPI7) is connected to the generator. A PicoScope (PS4824) is connected to the generator for the time-domain measurement. The PicoScope input is terminated with a $50 \Omega$ load to make sure it behaves the same as the test receiver.

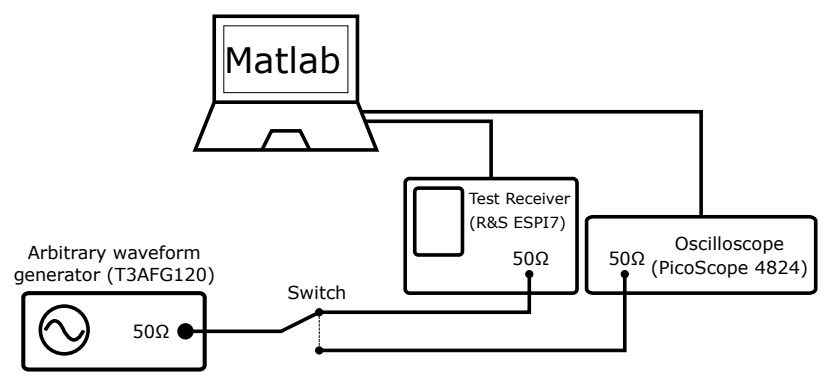

Fig. 5: Measurement setup for comparing dwell time estimation of a traditional EMI test receiver and a low cost oscilloscope

It is important to have a good test signal to show the effect of dwell time on the resulting power that is measured at a particular frequency with different types of detectors, for which on-off switching will be used. Apart from this an interference that is varied smoothly from zero to full power and back will also be used to emphasize on the effect of the resolution bandwidth (RBW) of the receiver. Table II gives a summary of the used parameters for the test signals.

At $100 \mathrm{kHz}$ it is expected that the minimum found dwell time will be 1 second, such that a correct reading is always detected. It is also expected that the three frequency components around $101 \mathrm{kHz}$ will be detected as a single peak appearing and fading with a $3 \mathrm{~Hz}$ frequency. This is due to the fact that all three frequency components fit within the minimum 200 $\mathrm{Hz}$ RBW according to the CISPR-16-1-1 [16].

\section{TABLE II: PARAMETERS OF THE TEST SIGNALS}

\begin{tabular}{|l|l|l|}
\hline Parameter & On-off interference & Smooth varying \\
\hline Disturbance frequency & $100 \mathrm{kHz}$ & $101 \mathrm{kHz}$ \\
Switching frequency & $1 \mathrm{~Hz}$ & $3 \mathrm{~Hz}$ \\
Duty cycle & $50 \%$ & $-\%$ \\
Disturbance Amplitude & $125 \mathrm{mV}$ & $125 \mathrm{mV}$ \\
Generator Output Impedance & $50 \Omega$ & $50 \Omega$ \\
EMI receiver input impedance & $50 \Omega$ & $50 \Omega$ \\
Picoscope input impedance & $50 \Omega$ & $50 \Omega$ \\
Resolution Bandwidth Receiver & $200 \mathrm{~Hz}$ & $200 \mathrm{~Hz}$ \\
\hline
\end{tabular}

\section{EXPERIMENTAL RESULTS}

Two different techniques are used to determine the dwell times. A conventional EMI test receiver uses a lot of measurements and takes a long time to determine the dwell
TABLE III: MEASUREMENT PARAMETERS DEFINED BY NRE01

\begin{tabular}{|c|c|c|c|}
\hline Frequency Range & Bandwidth & Step Size & Measuring Time \\
\hline $30 \mathrm{~Hz}-1 \mathrm{kHz}$ & $10 \mathrm{~Hz}$ & $5 \mathrm{~Hz}$ & 2 seconds \\
$1 \mathrm{kHz}-10 \mathrm{kHz}$ & $100 \mathrm{~Hz}$ & $50 \mathrm{~Hz}$ & 0.2 seconds \\
$10 \mathrm{kHz}-100 \mathrm{kHz}$ & $1 \mathrm{kHz}$ & $500 \mathrm{~Hz}$ & 0.02 seconds \\
\hline
\end{tabular}

time at only one frequency bin. If the dwell time would not be determined beforehand one would have to measure every frequency bin for the maximum measurement time to accurately estimate a disturbance. For a NRE01 test these minimum measurement times are defined and this would result in extremely long measurement times, since this test requires many bins throughout the specified frequency range, as seen in Table III. The time-domain technique determines all dwell times at every significant frequency component simultaneously via one measurement using only a multichannel low cost digitizer in conjunction with signal processing on a PC. This information can then be used to save time at the electromagnetic compatibility (EMC) compliance test.

\section{A. Determining Dwell Time Using the ESPI7 at $100 \mathrm{kHz}$}

To determine the dwell time using the traditional test receiver multiple measurements had to be performed for different measurement times at a single frequency. At $100 \mathrm{kHz}$, 100 measurements have been performed for the following measurement times, $50 \mathrm{~ms}, 100 \mathrm{~ms}, 500 \mathrm{~ms}, 1 \mathrm{~s}, 2 \mathrm{~s}$. Different expectations can be written down for the different detectors, since we know that the $\mathrm{CW}$ interference in this frequency bin is being switched on and off with a switch frequency of $1 \mathrm{~Hz}$ with a duty cycle of $50 \%$.

1) Peak detector: The expected result for the peak measurements, for measurement times far below the dwell time, is that half of the measurement points are at the maximum value and half of them are at $0 \mathrm{~V}$, which gives a probability of intercept of 0.5. As the measurement time increases the number of measurements at the maximum also increases until the measurement time reaches half of the dwell time, since the interfering $\mathrm{CW}$ is being switched on and off with a duty cycle of $50 \%$. This effect can be seen in Fig. 6 .

2) Average detector: For the average detector a uniform distribution of values is expected for exactly half of the dwell time, this can be clearly seen in Fig. 7. At 500 ms almost every value between the maximum and zero can be found, and only one specific value can be seen at a measurement time of $1 \mathrm{~s}$.

\section{B. Determining Dwell Time Using the ESPI7 at $101 \mathrm{kHz}$}

The same measurements have been repeated at the $101 \mathrm{kHz}$ frequency components where a minimum dwell time of $500 \mathrm{~ms}$ has been found due to the predefined measurement times with the ESPI7. This $500 \mathrm{~ms}$ corresponds to the expected value, which would have been $0.33 \mathrm{~ms}$ if any measurement time for the ESPI7 could have been taken. It is also important to emphasize on the fact that if the RBW would have been smaller than the frequency of the time variation it would 


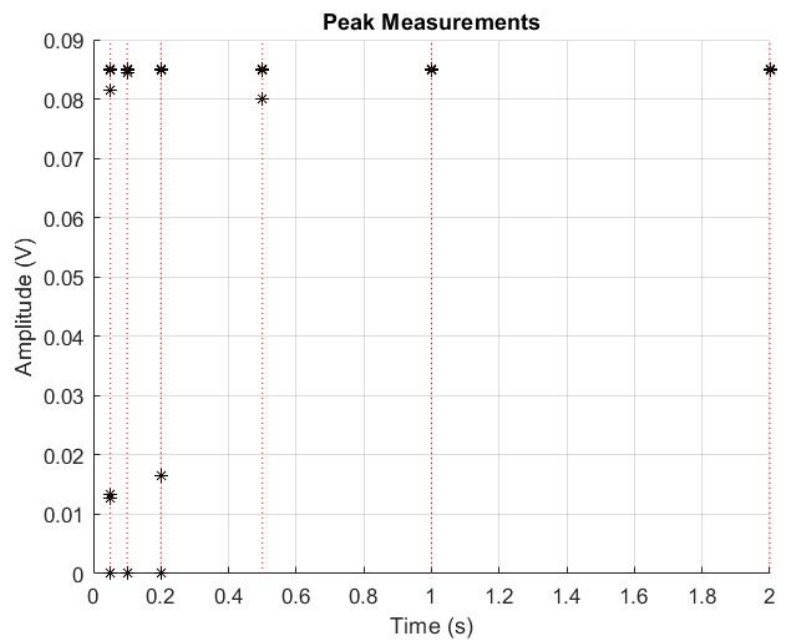

Fig. 6: ESPI7 peak measurement at $100 \mathrm{kHz}$ with a timevarying on-off interference source

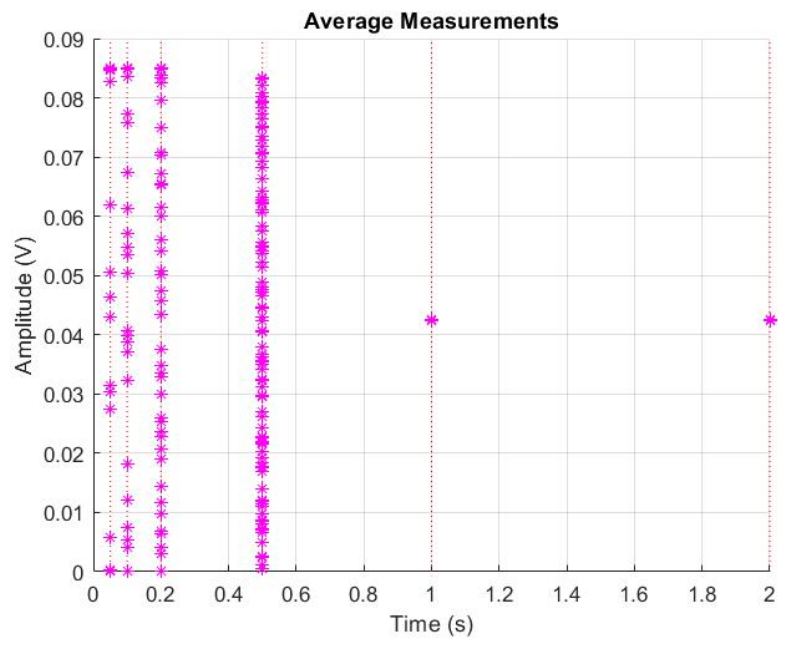

Fig. 7: ESPI7 average measurement at $100 \mathrm{kHz}$ with a timevarying on-off interference source

perceive the signal as three individual continuous waves, instead of the clearly time varying signal.

\section{Determining Dwell Time Using STFFT}

It is important to emphasize that conventional EMI test receiver measurements, to find the dwell time, have been performed at single frequency bins, with the minimum resolution bandwidth of $200 \mathrm{~Hz}$. The time-domain measurement via the low cost digitizer however performed the same measurement simultaneously over the entire frequency band only limited by the sampling rate, $0-500 \mathrm{kHz}$, finding all the significant dwell times, within frequency bins of $200 \mathrm{~Hz}$, as to mimic the test receiver. This is realized by taking the FFT of every time slice at every frequency bin of the STFFT seen in Fig. 1. From each FFT of the time slices of the frequency bins, the most significant slowest frequency is chosen, since this slow frequency results in a longest dwell time and therefore also incorporates the shorter dwell times. This choice can be seen as taking the worst case scenario. Only dwell times for frequency bins with a significant power are calculated, and the results can be seen in Fig. 8 .

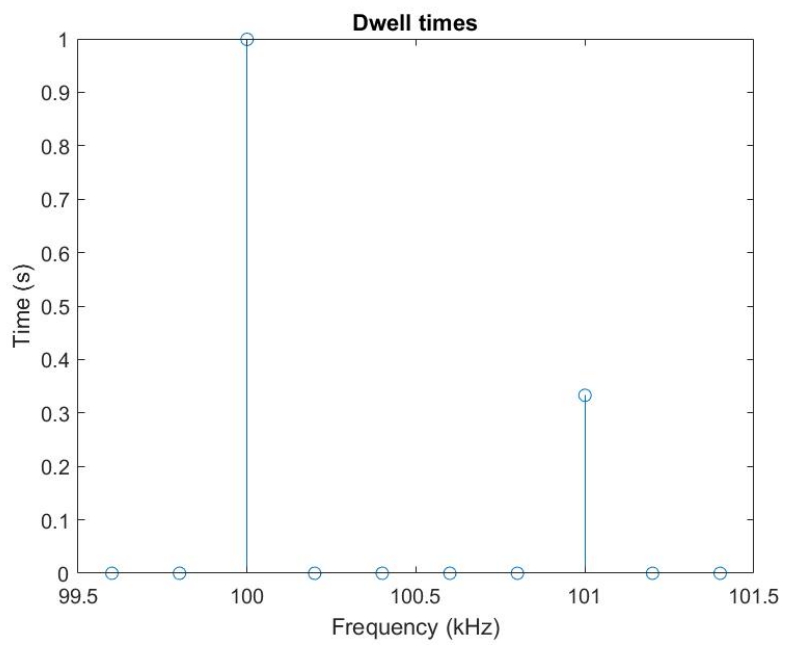

Fig. 8: Time-domain result for determining dwell times

As a practical example a switch mode power supply with a relatively slow switching load was used. The radiated EMI emission was measured via a loop antenna compliant with NRE01. For determining the dwell times the measured timedomain signal is used straight from the same low cost digitizer as the one described in Fig. 5. The spectrogram created from this time-domain signal can be seen in Fig. 9.

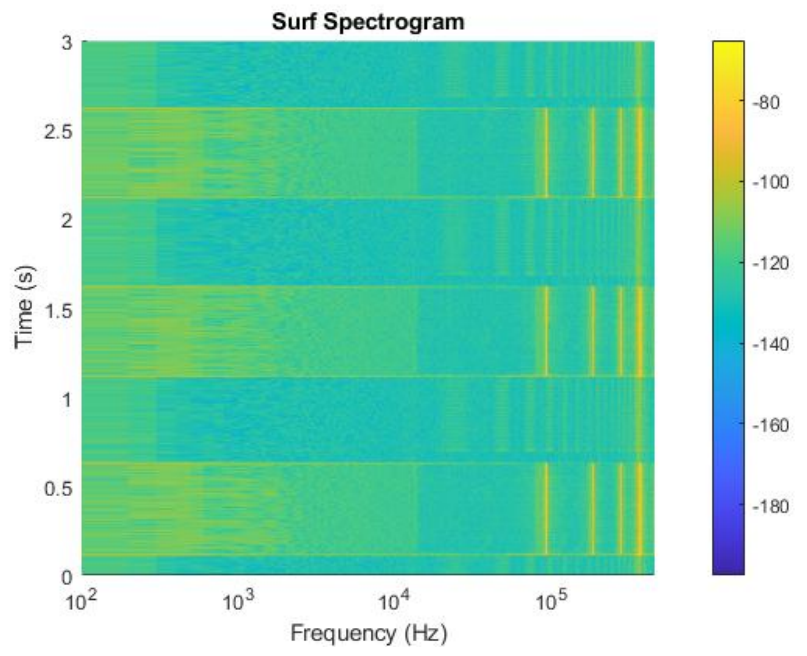

Fig. 9: Spectrogram of a switch mode power supply with a slow switching load

From Fig. 9 a clear time varying interference can be seen at around $90 \mathrm{kHz}$ and its multiples. The DSP on a PC is used to determine the dwell times of these time varying interferences. The dwell times for the entire frequency range are determined simultaneously, but only the dwell times at 
frequencies with significant power are shown. This result can be seen in Fig. 10 where the dwell times can be clearly seen at $90 \mathrm{kHz}$ and its multiples. If these measurements would have otherwise have been performed with the conventional EMI receiver it would have taken a significantly longer time to get limited information. Now with one $3 \mathrm{~s}$ measurement the entire frequency range has been accounted for, after which DSP can take care of the rest, with respect to analysis with certain bin sizes according to the NRE01 standard for example. This information would mean only the bins around multiples of $90 \mathrm{kHz}$ would need to be measured for at least 1 second, while all the other bins can be quickly swept through with a really low dwell time. For large equipment this would save hours of measurement times.

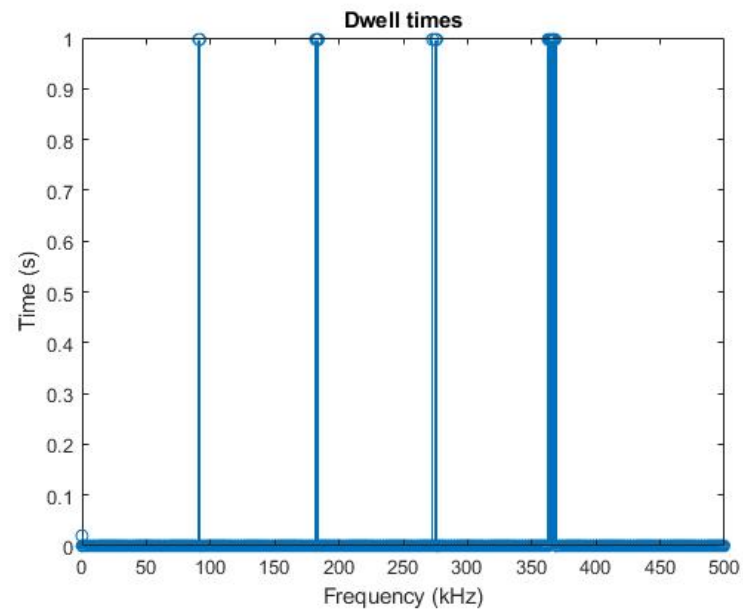

Fig. 10: Dwell times of a switch mode power supply with a slow switching load

\section{CONCLUSiON}

Utilizing time-domain EMI measurements with a low cost digitzer via DSP software on a PC to estimate the dwell times for a conventional EMI receiver has been investigated. These dwell times are calculated by taking FFTs of every time slice for every frequency bin from the spectrogram created via the STFFT. The found dwell time at a single frequency has been verified by extensive and time consuming measurements via the traditional receiver. With the time-domain measurements, all dwell times at every significant frequency component are calculated simultaneously via one measurement using only a multichannel low cost digitizer in conjunction with signal processing on a PC. This information can be used to optimize measurements with a conventional super-heterodyne frequency band stepping EMI test receiver, like the ESPI7, to save time while still complying to the standards. This optimization reduces a lot of the detrimental measurement times otherwise needed in the expensive test labs. Several options with the DSP have been described such as mimicking the detector bandwidth and the effects this has on the perception of time-domain variation of frequency components of the signal. Mimicking of the test receivers, however, induces constraints on the timedomain data. The fact is that with time-domain measurements all the necessary information is measured, but when mimicking the test receivers, to comply with the standards, a lot of useful information is discarded as a result of limitations from the past. These time-domain measurement also incorporate much more information while being significantly faster, especially when one compares them to the conventional measurements at the lower frequency ranges, $30 \mathrm{~Hz}-150 \mathrm{kHz}$, for which NRE01 needs extremely long measurement times stepping through the entire frequency band. These time-domain measurements can be realized with relatively low cost digitizer which can also function on multiple channels simultaneously while still outperforming conventional receivers. For the future a complete change to time-domain measurements incorporated within the standards should be the way to go.

\section{REFERENCES}

[1] E. Puri and M. Monti, "Pitfalls in Measuring Discontinuous Disturbances with Latest Click Analysers," Emc2016, pp. 1-6, 2016.

[2] S. M. A. Solar and T. Ag, "Electromagnetic ( Environmental ) Compatibility," no. January, pp. 1-8, 2011.

[3] I. Setiawan, C. Keyer, F. Buesink, and F. Leferink, "Time-frequency diversity for solving e deadlock in defining interference levels in power lines," IEEE International Symposium on Electromagnetic Compatibility, vol. 2016-Novem, pp. 364-369, 2016.

[4] T. Karaca, B. Deutschmann, and G. Winkler, "EMI-receiver simulation model with quasi-peak detector," IEEE International Symposium on Electromagnetic Compatibility, vol. 2015-Septm, pp. 891-896, 2015.

[5] I. Setiawan, N. Moonen, F. Buesink, and F. Leferink, "Efficient Magnetic Field Measurements," 2017.

[6] T. Hartman, N. Moonen, B. ten Have, and F. Leferink, "Fast magnetic emission tests for continuous measurements around an equipment under test," in 2019 ESA Workshop on Aerospace EMC (Aerospace EMC), May 2019, pp. 1-5.

[7] M. Pous, M. Azpúrua, and F. Silva, "Benefits of Full Time-Domain EMI Measurements for Large Fixed Installation,” pp. 514-519, 2016.

[8] E. Puri and M. Monti, "Hidden Aspects in CISPR 16-1-1 Full Compliant Fast Fourier Transform EMI Receivers," pp. 34-39, 2016.

[9] E. Puri and M. Monti, "The Importance of Overload Revealing in EMI Receivers," 2017.

[10] M. A. Azpúrua, J. A. Oliva, M. Pous, and F. Silva, "Robust Extreme Value Estimation for Full Time-Domain EMI measurements," 2017 International Symposium on Electromagnetic Compatibility - EMC EUROPE 2017, EMC Europe 2017, pp. 4-9, 2017.

[11] M. A. Azpúrua, M. Pous, J. A. Oliva, B. Pinter, M. Hudlička, and F. Silva, "Waveform approach for assessing conformity of cispr 161-1 measuring receivers," IEEE Transactions on Instrumentation and Measurement, vol. 67, no. 5, pp. 1187-1198, 2018.

[12] T. Hartman, N. Moonen, and F. Leferink, "Evaluation of Multichannel Synchronous Conducted TDEMI Measurements for High Voltage Power Electronics," IEEE International Symposium on Electromagnetic Compatibility, vol. 2018-Augus, pp. 839-843, 2018.

[13] T. Hartman, N. Moonen, and F. Leferink, "Direct Sampling in Multichannel Synchronous TDEMI Measurements," 2018 IEEE 4th Global Electromagnetic Compatibility Conference (GEMCCON), pp. 1-5, 2019.

[14] Rohde \& Schwarz, "Operating Manual EMI Test Receiver ESPI7,' Tech. Rep.

[15] Teledyne Lecroy, “Operating Manual Teledyne Lecroy Function/Arbitrary Waveform Generator," Tech. Rep.

[16] International Electrotechnical Commission, "CISPR 16-1-1 Radio disturbance and immunity measuring apparatus - Measuring apparatus," Tech. Rep. 